\title{
Questionários de qualidade de vida relacionada à saúde na Doença Pulmonar Obstrutiva Crônica (DPOC): revisão de literatura
}

\section{Questionnaire on quality of life related to Chronic Obstrutctive Pulmonary Disease (COPD): literature review}

\section{Ana Cláudia Costa Carneiro', Marina Santos Costa', Daniel Machado Ferreira Costa ${ }^{2}$, Laís Costa Carneiro ${ }^{3}$, Aquiles Camelier ${ }^{4}$}

'Autora para correspondência. Escola Bahiana de Medicina e Saúde Pública (EBMSP), Salvador, Bahia, Brasil. carneiroacc@gmail.com ${ }^{2}$ Escola Bahiana de Medicina e Saúde Pública (EBMSP), Salvador, Bahia, Brasil. marina.costa94@gmail.com; danmfcosta@gmail.com 3Universidade Federal da Bahia (UFBa), Salvador, Bahia, Brasil. laisccarneiro01@gmail.com ${ }^{4}$ Escola Bahiana de Medicina e Saúde Pública (EBMSP); Universidade do Estado da Bahia (Uneb), Salvador, Bahia, Brasil. aquilescamelier@yahoo.com.br

Resumo I INTRODUÇÃO: Pacientes com DPOC geralmente experimentam uma diminuição na qualidade de vida relacionada à saúde (QVRS) a partir da percepção própria de que se sentem limitados em algum aspecto de suas vidas. OBJETIVO: Analisar as produções científicas que abordam o uso de questionários de qualidade de vida para acompanhar evolução de doença em portadores de Doenças Pulmonares Obstrutivas Crônicas. MÉTODOS E MATERIAIS: Revisão Não Sistemática da literatura incluindo artigos nos idiomas inglês, português e espanhol, a partir das bases de dados Medline, SciELO e Lilacs, no período de 1998 a 2017, além de livros técnicos que versam sobre o assunto. Foram utilizados os termos: "chronic obstructive pulmonary disease", "quality of life", "specific health-related quality of life questionnaires". RESULTADOS/SÍNTESE DOS DADOS: Os 39 artigos encontrados foram organizados em duas seções, a saber: estudos de validação de questionários de qualidade de vida $(n=22)$ e estudos de comparação entre instrumentos genéricos e específicos para avaliação de qualidade de vida $(n=17)$. CONCLUSÕES: A avaliação da qualidade de vida deve ser incorporada ao seguimento clínico, uma vez que a doença crônica repercute nas diversas dimensões da vida dos pacientes. Os questionários específicos de qualidade de vida dão uma melhor dimensão das condições de saúde no portador de DPOC no que se refere a sua relação com a doença.

PALAVRAS-CHAVE: Doença Pulmonar Obstrutiva Crônica. Qualidade de vida. Questionários. Revisão.

\begin{abstract}
I INTRODUCTION: Patients with Chronic Obstructive Pulmonary Disease (COPD) usually experience a decrease in quality of life related to health (QLRH) from their perception of their own limitations in some aspects of their lives. OBJECTIVE: To analyze the scientific production which tackle the use of questionnaires on quality of life in order to follow the disease evolution in subjects with COPD. METHODS AND MATERIALS: NonSystematic Review of the literature including articles in English, Portuguese and Spanish, using Medline, SciELO and Lilacs data bases, from 1998 to 2017 , as well as technical books on the theme. The terms used were "chronic obstructive pulmonary disease", "quality of life", "specific health-related quality of life questionnaires". RESULTS/ DATA SYNTHESIS: The 39 articles found were organized into two sections as follows: studies on validation of quality of life questionnaires $(n=22)$ and studies of comparison between generic and specific instruments for quality of life evaluation $(n=17)$. CONCLUSIONS: The evaluation of quality of life for patients with COPD must be incorporated to the clinical segment, as the chronic disease influences several dimensions of patients' lives. The specific questionnaires on quality of life give a better dimension of the health conditions of patients with COPD regarding their relationship with the disease.
\end{abstract}

KEY-WORDS: Chronic Obstructive Pulmonary Disease. Quality of life. Questionnaires: Review. 
Introdução

A Doença Pulmonar Obstrutiva Crônica (DPOC), na versão de 2017 do documento da Iniciativa Global para a Doença Obstrutiva Crônica (Global Initiative for Obstructive Lung Disease - GOLD) é conceituada como uma doença comum, prevenível e tratável, e caracterizada por sintomas respiratórios persistentes e limitação ao fluxo aéreo, que decorre de anormalidades nas vias aéreas e nos alvéolos causadas por exposição significativas a partículas ou gases nocivos'. Segundo dados da Organização Mundial da Saúde (OMS), a DPOC é uma das doenças mais prevalentes em todo o mundo e está prevista para ser a terceira causa de morte até 2030. No Brasil, as internações decorrentes desta doença representaram um número na ordem de $170 \mathrm{mil}$ admissões (DATASUS, 2008). O número de óbitos por DPOC variou em torno de 33.100 mortes anuais, de 2000 a 2005 (DATASUS, 2008). A DPOC é responsável por um enorme custo financeiro, promovendo gastos da ordem de US $\$ 1.522,00$ por paciente por ano 2 .

Pacientes com DPOC geralmente experimentam uma diminuição na qualidade de vida relacionada à saúde (QVRS) a partir da percepção própria de que se sentem limitados em algum aspecto de suas vidas $^{3}$. Sintomas comuns na DPOC como tosse, produção de expectoração e, principalmente, dispneia ou intolerância a esforços, bem como exacerbações agudas da doença e comorbidades que são frequentemente associadas em pacientes com DPOC, contribuem para a redução da percepção da QVRS e para a gravidade geral da doença ${ }^{4}$.

O conceito subjetivo e multidimensional de Qualidade de Vida (QV) consolidou-se entre os estudiosos na década de 90 e, desde então, identificar, mensurar e avaliar os domínios afetados pela doença tem sido a proposta de diversos estudos com delineamentos qualitativos e quantitativos ${ }^{5}$. Entretanto, enquanto que o termo "Qualidade de Vida" encontra-se associado na literatura a um rótulo genérico referente a uma quantificação no desempenho físico ou a variáveis psicossociais em geral ( o que, por sua vez, remete-se ao amplo conceito de "Saúde" proposto pela OMS - "um estado de completo bem-estar físico, mental e social e não somente ausência de afeções e enfermidades"), o termo "Qualidade de
Vida Relacionada à Saúde ou QVRS" ( também denominado de "Estado de Saúde") é definido como a quantificação ou avaliação do impacto específico de uma doença ou condição patológica na percepção da qualidade de vida em um indivíduo ${ }^{6,7}$.

\section{Métodos}

Foram realizadas leituras de livros textos referentes ao tema QVRS e desenvolvida busca de estudos nas bases de dados Medline (PubMed), Scielo e LILACS por meio dos termos e operadores boleanos (chronic obstructive pulmonary disease [Title]) and (quality of life) and (specific health-related quality of life questionnaires), usados para identificar títulos e resumos de artigos originais e de revisão publicados entre os anos de 1998 e 2017. A busca limitou-se a artigos em língua inglesa, espanhola ou portuguesa. Foram selecionados os artigos mais relevantes para atualização do uso de questionários de qualidade de vida em acompanhamento do portador de DPOC.

Para o entendimento das definições relacionadas à qualidade de vida, faz-se necessário explanar sobre definições relacionadas ao tema:

\section{O Conceito de saúde}

Ao ser constituída em 1946, final da segunda guerra, a Organização Mundial da Saúde (OMS/WHO) definiu a saúde como sendo "um estado de completo bem-estar físico, mental e social, que não consiste apenas na ausência de doença". O conceito foi divulgado na carta de princípios de 7 de abril de 1948 (desde então estabeleceu-se o Dia Mundial da Saúde). Considera ainda que, gozar do meIhor estado de saúde que é possível atingir, constitui um dos direitos fundamentais de todo o ser humano, sem distinção de raça, de religião, de credo político, de condição econômica ou social. Afirma ainda que saúde de todos os povos é essencial para conseguir a paz e a segurança e depende da mais estreita cooperação dos indivíduos e dos Estados ${ }^{8}$.

Em 1974, Marc Lalonde, titular do Ministério da Saúde e do Bem-estar do Canadá, defendeu o conceito de que o campo da saúde abrange: a 
biologia humana (herança genética e os processos biológicos inerentes à vida); o meio ambiente; o estilo de vida (tabagismo, etilismo, sedentarismo); a organização da assistência à saúde (a ser promovido pelo estado)?.

No Brasil, nossa Constituição Federal de 1988, em seu Artigo 196, diz que: "A saúde é direito de todos e dever do Estado, garantida mediante políticas sociais e econômicas que visem à redução do risco de doença e de outros agravos e ao acesso universal e igualitário às ações e serviços para a promoção, proteção e recuperação". ${ }^{10}$

\section{O conceito de satisfação}

O termo satisfação tem sua origem no latim "satisfactione" e se refere ao ato ou efeito de satisfazer, significando saciedade ou sentimento de bem-estar que se manifesta nas pessoas, como resultado da realização do esperado ou desejado, sob a forma de alegria, contentamento, agrado e aprazimento. Assim sendo, fica patente que se trata de uma sensação subjetiva e dinâmica, com diferentes significados, em diferentes contextos e com forte valor cultural. A partir de 2000, a OMS passou a utilizar em pesquisas de avaliação em saúde o conceito de responsividade dos sistemas de saúde, em contraposição ao de satisfação, em função de valorizar o impacto da presença das doenças e seus tratamentos nas pessoas, em detrimento dos aspectos culturais ou econômicos. Pesquisas sobre responsividade compreendem duas finalidades: a primeira é medir o que acontece quando as pessoas interagem com o sistema de saúde, 0 que implica coletar dados sobre o comportamento, evento ou ação do sistema. A segunda é medir como as pessoas atendidas pelo sistema de saúde percebem e avaliam o que acontece ${ }^{11}$.

\section{Conceito de Qualidade de Vida (QV)}

O conceito de QV é muito abrangente, demandando, na tentativa de estabelecer um consenso, um envolvimento de profissionais de várias áreas do conhecimento (cientistas, filósofos, políticos). Existem citações de que o filósofo Sócrates já fazia referências ao conceito de qualidade de vida. ${ }^{12} \mathrm{O}$ termo QV é amplamente empregado no cotidiano e por profissionais no contexto da pesquisa científica em diferentes campos, incluindo a medicina ${ }^{13}$.
A investigação de $Q V$ engloba as perspectivas, objetivos e práticas diferenciadas, tais como demográfica, bioética, econômica, ambiental e saúde pública ${ }^{13}$.

\section{Conceito de qualidade de vida relacionado à saú- de (QVRS)}

A Qualidade de vida relacionada à saúde (QVRS) é definida pela OMS como "a percepção que $\circ$ indivíduo tem sobre a sua posição na vida e no contexto de sua cultura, de acordo com os sistemas de valores da sociedade em que vive e em relação aos seus objetivos, expectativas, padrões e preocupações". ${ }^{8}$ Quatro domínios são compreendidos nesta definição: físico, psicológico, social e ambiental. Este âmbito geral denota a dimensão que o conceito de QVRS deve abranger como indicador da patologia: o estado funcional (funções físicas, psicológicas e sociais) e percepções de saúde. Devem ser considerados nas avaliações e práticas especificas de cada área a influência da percepção do indivíduo em relação aos aspectos biopsicossociais, tornando o construto multidimensional e subjetivo ${ }^{13}$.

\section{Métodos de avaliação na QVRS na DPOC}

As medidas de avaliação da QVRS são classificadas em três tipos: instrumentos genéricos, específicos e modulares. Os primeiros avaliam conceitos gerais de saúde, não são específicos para idade, doenças ou tipos de tratamento, são utilizados para avaliar diferentes domínios de saúde em populações gerais. Ainda que possam perder a sensibilidade em detectar pequenas diferenças em algum aspecto específico da QVRS, eles têm a capacidade de possibilitar a comparação entre diferentes doenças. Os questionários específicos, por sua vez, avaliam conceitos de saúde especialmente delineados a uma determinada doença ou intervenção e possuem escores separados para cada domínio, agrupando os aspectos de saúde considerados no processo de avaliação ${ }^{3}$. Em geral, eles tem maior sensibilidade em detectar pequenas alterações da QVRS, mas não permitem comparações entre grupos de doenças com diferentes aspectos da fisiopatologia. Os modulares combinam os aspectos genéricos e específicos da doença a ser avaliada ${ }^{13}$. 
São propriedades das medidas que devem ser observadas quando estamos utilizando questionário: 1) Confiabilidade- representada pela reprodutibilidade teste/reteste, quando pelo mesmo observador (reprodutibilidade intra observador) ou por observadores diferentes (inter observador). É avaliada estatisticamente por coeficientes de correlação. 2) Validade, isto é a capacidade de um instrumento medir ou avaliar aquilo a que se propõe. Avaliada estatisticamente por coeficientes de correlação Pearson. 3) Responsividade - que é a capacidade que um índice apresenta de detectar uma mudança, caso ocorra, em um determinado período de tempo ${ }^{13}$. Ainda dentro do conceito da responsividade, deve ser levado em conta a magnitude desta mudança (no caso dos questionários, a mudança na pontuação), e se esta variação na pontuação é suficiente para provocar uma diferente percepção no indivíduo avaliado, também chamado de mínima diferença clinicamente importante $(\mathrm{MDCl})^{14}$.

Os questionários de qualidade de vida também devem ser testados quanto às propriedades estudadas de consistência interna, reprodutibilidade teste/reteste e validade de critério. Nestes estudos de reprodutibilidade, pode-se determinar a variação na pontuação destes questionários referentes a mera variabilidade de respostas, não atribuídas à uma mudança de percepção da QVRS. Ao determinar esta variabilidade espontânea pode-se ter uma adequada ferramenta para o cálculo amostral de estudos futuros, além de serem estudos que auxiliam na determinação da $\mathrm{MDCl}^{15}$. Após esta avaliação as propriedades psicométricas da versão utilizada do questionário são consideradas suficientes ou não ${ }^{16}$.

\section{Resultados}

Foram selecionados 39 artigos e organizados em duas seções, a saber: estudos de validação de questionários de qualidade de vida $(n=22)$ e estudos de comparação entre instrumentos genéricos e específicos para avaliação de qualidade de vida $(n=17)$.

A seleção do instrumento a ser utilizado para avaliar QVRS demanda muito cuidado, pois deve ser observado o tipo de comparação a ser realizada, a heterogeneidade dos sujeitos avaliados, assim como a capacidade de compreender e preencher questionários e, além disso, o questionário tem que ter condições viáveis de recolher os dados em situações da prática clínica habitual. Este cuidado necessita ser maior ainda em ensaios clínicos controlados e deve ter em conta pressões de tempo e variabilidade na capacidade dos doentes de responder. A seleção dos instrumentos deve centrar-se na utilização de medidas específicas para cada doença, reservando as medidas genéricas para a identificação dos efeitos mais amplos das co-morbilidades ${ }^{17}$.

A mensuração da QVRS em portadores de doença respiratória crônica é agora uma forma habitual de avaliação de resultado de intervenções terapêuticas. Estudos com questionários têm uma dupla avaliação: a) observação da significância encontrada na mudança experimentada por pacientes em sua qualidade de vida (a significância estatística das diferenças observadas são analisados) e b) avaliação do possível significado clínico atribuído a esta mudança na qualidade de vida ${ }^{18}$.

A ATS (American Thoracic Society), ao discorrer sobre qualidade de vida em DPOC, considera que esta foi a primeira doença respiratória a ser completamente estudada com instrumentos de qualidade de vida relacionados à saúde e que, com eles, o efeito da DPOC na vida dos indivíduos foi bem caracterizado. Muitos estudos de qualidade de vida empregaram instrumentos genéricos e instrumentos de qualidade de vida relacionados à saúde para determinar a eficácia de tratamentos e programas de reabilitação sobre a vida de pacientes com esta doença. ${ }^{19}$

Vários instrumentos de qualidade de vida relacionados com a saúde, bem validados, também foram desenvolvidos para indivíduos com DPOC. Podem ser listados: o Dartmouth COOP Charts -medida genérica de estado funcional projetada para uso clínico em atenção primária; ○ EuroQol ou EQ-5D- uma medida genérica usada para caracterizar estados de saúde atuais, que consiste em 5 domínios e uma escala analógica visual; Functional Performance Inventory FPI -uma medida de auto relato dos próprios pacientes com DPOC do seu desempenho funcional, desenvolvida a partir de uma estrutura analítica ex- 
plícita. $O$ desempenho é definido como as atividades diárias que os pacientes fazem para atender às necessidades básicas, cumprir os papéis usuais e manter sua saúde e bem-estar; Measure Your Medical Outcome Profile MYMOP-é um instrumento simples projetado para autoadministração para uso em um ambiente de clínica geral; Nottingham Health Profile NHP -medida genérica de qualidade de vida relacionada à saúde. $O$ instrumento é utilizado para avaliar distúrbios percebidos em várias populações; Quality of Well-being Scale QWB - questionário de qualidade de vida de saúde geral, administrado pelo entrevistador, que mede sintomas, mobilidade, atividade física e atividade social. Pontuações podem ser traduzidas em avaliação econômica para estudos de custo-efetividade ou qualidade de anos de vida ajustados; SF-1 2 Health Survey e SF-12v2 Health Surve-O SF-12v2 TM Health Survey é um subconjunto de 12 itens do SF-36v2 TM que mede os mesmos oito domínios de saúde. É uma medida breve e confiável do estado geral de saúde. É útil em grandes inquéritos à saúde da população e tem sido amplamente utilizado como uma ferramenta de triagem; MOS 36-Item Short Form Health Survey MOS SF-36 - medida genérica de qualidade de vida relacionada à saúde. $O$ instrumento é amplamente utilizado para avaliar a QVRS em várias populações; Sickness Impact Profile SIP- medida genérica usada para avaliar o impacto da doença no funcionamento físico e emocional. Os pacientes são convidados a responder aos itens referentes a como eles estão naquele dia. A medida também tem sido utilizada em pacientes com DPOC e asma; World Health Organization Quality of Life assessment instrument WHOQOL-100 - é uma medida genérica, preenchida pelo paciente, que foi desenvolvida simultaneamente em 15 locais em todo o mundo. É focada em torno da definição de qualidade de vida defendida pela OMS, que inclui a cultura e o contexto que influenciam a percepção de saúde de um indivíduo; Airways Questionnaire AQ-20; AQ-30 é um instrumento específico projetado para medir o estado de saúde em asma e DPOC; Breathing Problem-Based Quality of Life Questionnaire (BP-QOL), Chronic Obstructive Pulmonary Disease Activity Rating Scale CARS - instrumento específico da doença projetado para medir a atividade relacionada à vida. Desenvolvido para uso em pacientes com doença pulmonar obstrutiva crônica; Chronic Respiratory Disease Questionnaire CRQ - é um questionário administrado por entrevistador que mede aspectos físicos e emocionais da doença respiratória crônica; Pulmonary Functional Status \& Dyspnea Questionnaire- PFSDQ -avalia estado funcional e dispnéia em pacientes com doenças pulmonares, é auto administrado; Pulmonary Functional Status Scale-PFSS - é um instrumento de avaliação funcional para uso em pacientes adultos com doenças pulmonares; Quality-of-Life for Respiratory Illness Questionnaire QOL-RIQ - é uma medida de qualidade de vida especifica, projetada para pacientes com obstrução reversível e fixa das vias aéreas. Os pacientes são questionados quanto a um problema, divididos em cada item quanto a sua evolução no último ano; St. George's Respiratory Questionnaire SGRQ- é um instrumento específico para doenças respiratórias, projetado para medir o impacto na saúde geral, na vida diária e no bem-estar percebido pelo paciente. Desenvolvido para uso em portadores de obstrução fixa e reversível das vias aéreas; Seattle Obstructive Lung Disease Questionnaire -é um breve questionário auto administrado utilizando um computador, projetado para medir a função física, função emocional, habilidades de enfrentamento e satisfação quanto ao tratamento aplicado em pacientes com DPOC; Teste de Avaliação da DPOC CAT- é um questionário curto e simples para monitorar o acompanhamento a longo prazo da DPOC. Dirigido à prática de cuidados primários. Validado em 3 estudos internacionais. É composto de 8 itens, e escala de escala de seis pontos, bom para detectar as exacerbações. Auto-administrado. Disponível em muitas traduções, embora nem todss validadas. Escores mais altos representam pior estado de saúde; Questionário Clinico de DPOC CCQ - detecta estados leves, moderados e graves de DPOC. Mede capacidades funcionais e mentais, bem como sintomas. específicos da DPOC, é auto-administrado , tem 10 itens sobre os sintomas da semana anterior é fácil de aplicar. Também disponível em versão $24 \mathrm{~h}$. Prático e amplamente utilizado. Tem mais de 53 traduções: nem todas são validadas. Escores mais altos representam pior estado de saúde ${ }^{19}$.

Os instrumentos de utilidade genérica mais comumente usados em doenças pulmonares são o EQ-5D questionário EuroQol em cinco dimensões, o Health Utility Index (HUI) e o 15D quinze dimensional, também já foram testados na DPOC além do Questionário de 36 itens (SF-36), do Perfil de Impacto de 
Doença (SIP), e do Perfil de Saúde de Nottingham $(\mathrm{NHP})^{20}$.

Vários autores buscaram uma comparação entre questionários de qualidade de vida genéricos e específicos para a DPOC. Muitos destes questionários, tanto genéricos como os específicos, têm mostrado que satisfazem as propriedades psicométricas de confiabilidade, validade e sensibilidade e, por conseguinte, são adequados para estudos descritivos e / ou avaliação em doentes com asma e DPOC. Porém, os questionários específicos para doenças respiratórias, ao contrário de genéricos, foram projetados a partir dos sintomas, limitações e distúrbios da vida diária que ocorrem na asma e no DPOC a fim de otimizar as propriedades do instrumento $e$, em especial, a sensibilidade às alterações. Harper e colaboradores avaliaram a confiabilidade e a validade de dois questionários específicos, ○ $S G R Q$ e $\circ C R Q$, e de 2 genéricos, SF-36 e EuroQoL, e observaram que os instrumentos específicos foram mais sensíveis à alterações no estado clínico. Entretanto, o questionário genérico SF-36 foi melhor para identificar as comorbidades $^{21}$. Da mesma forma, Desikan e col. em estudo de pacientes com DPOC, mostraram que o SF-36 é mais relacionado com a identificação ou reconhecimento, pelo paciente, da necessidade de procurar atendimento nos serviços de saúde, do que os questionários específicos $S G R Q$ e $C R Q^{18}$.

Tsukino, utilizando ○ Chronic Respiratory Disease Questionnaire (CRQ) como questionário especifico e - Perfil de Saúde Nottingham (NHP) como medida genérica de QVRS, concluiu que em pacientes com diagnóstico recente de DPOC, tanto o NHP como - CRQ foram capazes de detectar alterações na QVRS associadas a intervenções médicas eficazes. A influência das alterações na limitação do fluxo aéreo sobre a QVRS foi fraca neste estudo ${ }^{22}$.

A avaliação de qualidade de vida em pacientes com doenças respiratórias crônicas é um bom indicador da gravidade da doença, no caso da DPOC, e tem- se mostrado significativamente relacionada com a frequência de exacerbações da doença. Es- tudo utilizando o $S G R Q$, demonstrou que a QVRS é pior em pacientes com exacerbações mais frequentes, e que as exacerbações sucessivas limitam a qualidade de vida ${ }^{23}$. Sendo assim, controlar as exacerbações pode retardar a deterioração progressiva da saúde. Há referência na literatura de que a qualidade de vida de pacientes com DPOC, medida pelo $S G R Q$, pode ser um indicador independente de mortalidade, sendo um bom preditor de evolução da doença ${ }^{24}$.

No artigo de Sullivan que versa sobre orientações críticas para a leitura de trabalhos sobre qualidade de vida, é citado um relatório que demostrou, em pacientes com asma e DPOC, como uma revisão baseada em evidências poderia ser concluída com QVRS como uma das medidas de desfecho primário. Hoje há padronização para utilizar o resultado da eficácia do tratamento a partir de relato do paciente, tratando-se os dados com base nos critérios convencionais da medicina baseada em evidências ${ }^{25}$.

É consenso que a DPOC interfere na QVRS, no entanto há na literatura pouca concordância com relação a identificação dos fatores que contribuem para a QVD comprometida. Estudo realizado por Engström e colaboradores demonstrou que embora a função pulmonar tenha sido importante, a qualidade de vida relacionada à saúde foi significativamente modulada por três dos fatores analisados no estudo: teste da caminhada de 6 minutos, limitação relacionada à dispneia e escore de depressão ${ }^{26}$.

Em estudo realizado na atenção primaria em sete países europeus, utilizando o SGRQ-C, a pesquisa de saúde de forma curta (SF-12) e a escala de Fadiga de Avaliação Funcional da Doença Crônica (FACIT), observou-se que a pontuação total média para SGRQ foi de 44,7 $\pm 19,4$ mostrando comprometimento marcado da QVRS. Os resultados do SF12 e FACIT-F foram consistentes com os do SGRQ-C. Este grande estudo observacional mostrou que o estado de saúde é significativamente prejudicado em pacientes com DPOC de todas as gravidades, mesmo naqueles com obstrução leve das vias aéreas e que, dentro de cada estádio GOLD de severidade, 
há considerável heterogeneidade no comprometimento da QVRS entre os pacientes ${ }^{27}$.

Em uma grande coorte de pacientes com DPOC na Finlândia, foram comparados, quanto à sua aplicabilidade, os instrumentos AQ20 (questionário de vias aéreas 20 ) um questionário especifico, e o 15D (quinze dimensional), este último genérico. Os autores concluíram que os escores das questões correspondentes e dimensões de AQ20 e 15D, respectivamente, e as pontuações sumárias de ambos os instrumentos são comparáveis em termos de mensuração da QVRS em pacientes com DPOC. Considerando assim que seus dados suportam a validade convergente de 15D para medir a qualidade de vida na DPOC $^{20}$.

Em um trabalho de validação do $A Q 20$ em pacientes portadores de DPOC no Brasil, foi concluído que - AQ20 é um questionário reprodutível, de rápida aplicação, com boa correlação com ○ SGRQ, podendo ser utilizado para avaliação do estado de saúde em pacientes com DPOC, principalmente em situações em que o tempo disponível para análise da qualidade de vida seja reduzido28. Além disto, - AQ20 guardou, em um estudo ambulatorial com portadores de doenças pulmonares obstrutivas crônicas, um bom poder discriminatório em identificar indivíduos com diferentes percepções de QVRS e uma excelente acurácia através da análise da curva ROC em predizer a pontuação do $S G R Q$, com área sob a curva ROC igual a 0,91 (IC95\% 0,82-0,99; $\mathrm{p}<0,001)^{29}$. Adicionalmente, em um outro estudo também no Brasil, o AQ20 esteve significativamente associado a desfechos relevantes na DPOC, como o $\mathrm{VEF}_{1}$, a $\mathrm{SpO}_{2}$, o teste de caminhada de seis minutos, a dispneia e com $\circ$ índice multidimensional de mortalidade BODE (IMC, obstrução das vias aéreas, dispneia e capacidade de exercício) ${ }^{30}$. O AQ20 possui uma pontuação que varia de 0 a $100 \%$, e, quanto maior a pontuação, pior a qualidade de vida. Não existem valores de normalidade, até o presente momento, definidas para o $\mathrm{AQ} 20^{28}$.
O SGRQ foi validado para ○ Brasil inicialmente no ano de 2000, e teve sua versão brasileira atualizada em $2006^{31,32}$. O mesmo possui uma pontuação que varia de 0 a $100 \%$ (quanto maior a pontuação, pior a qualidade de vida, valores menores que $10 \%$ são considerados de QVRS normal para portadores da $D P O C^{33}$. A MDCl para $\circ S G R Q$ está definida em 4 unidades percentuais. Em um estudo que comparou ○ SGRQ (específico) com ○ SF-36(genérico) observou que $\circ$ primeiro demonstrou maior capacidade de discriminação entre os diferentes níveis de gravidade dos estágios da DPOC e está mais fortemente correlacionado com as medidas clínicas da DPOC do que as medidas genéricas de saúde. No entanto, as medidas genéricas destinam-se a captar aspectos mais amplos da saúde, e podem então trazer informação adicional sobre a QVRS que não está relacionada com a DPOC apenas ${ }^{20}$.

As medidas utilizadas nos questionários devem ser adequadas à questão abordada, serem sensíveis às alterações relevantes para os pacientes, e capazes de fornecer aos médicos escores significativos e aceitáveis. Os questionários que preencham estes esses atributos e que além disso sejam rápidos e fáceis de usar durante as consultas são eficazes. A incorporação de questionários como estes no processo de consulta possibilitará uma melhor tomada de decisão de parceria médico-paciente, ajudará o paciente a priorizar a necessidade de revisão nos cuidados primários de atenção à saúde e conduzirá ao manejo efetivo de pacientes com DPOC ${ }^{34}$.

Uma revisão realizada pela International Primary Care Respiratory Group (IPCRG) produziu um guia do usuário que revisa nove das mais de 42 ferramentas que medem a doença ou a experiência de bem-estar do paciente com DPOC. Incluiu ferramentas que medem o estado de saúde ou a qualidade de vida, bem como ferramentas que medem as características da DPOC, como dispneia e problemas respiratórios ${ }^{35}$. 
Tabela 1. International Primary Care Respiratory Group (IPCRG) modificada

\begin{tabular}{|l|l|l|l|l|l|l|}
\hline Tool & Validity/Reliability & Responsivity & $\begin{array}{l}\text { Primary Care } \\
\text { Population }\end{array}$ & $\begin{array}{l}\text { Practical } \\
\text { Easy to Apply }\end{array}$ & $\begin{array}{l}\text { Tested } \\
\text { practice }\end{array}$ & $\begin{array}{l}\text { Translated to } \\
\text { other } \\
\text { languages }\end{array}$ \\
\hline AQ20 & $\begin{array}{l}\text { Highly } \\
\text { recommended }\end{array}$ & Recommended & Recommended & $\begin{array}{l}\text { Highly } \\
\text { recommended }\end{array}$ & Good & Insufficient \\
\hline BPQ-S & Highly & Good & Recommended & Good & Insufficient & Insufficient \\
\hline CARS & Good & Insufficient & Insufficient & Good & Insufficient & Insufficient \\
\hline CAT & $\begin{array}{l}\text { Highly } \\
\text { recommended }\end{array}$ & Recommended & $\begin{array}{l}\text { Highly } \\
\text { recommended }\end{array}$ & Recommended & Good & Recommended \\
\hline CCQ & $\begin{array}{l}\text { Highly } \\
\text { recommended }\end{array}$ & $\begin{array}{l}\text { Highly } \\
\text { recommended }\end{array}$ & $\begin{array}{l}\text { Highly } \\
\text { recommended }\end{array}$ & $\begin{array}{l}\text { Highly } \\
\text { recommended }\end{array}$ & $\begin{array}{l}\text { Highly } \\
\text { recommended }\end{array}$ & $\begin{array}{l}\text { Highly } \\
\text { recommended }\end{array}$ \\
\hline CRQ & $\begin{array}{l}\text { recommended } \\
\text { recommed }\end{array}$ & Recommended & $\begin{array}{l}\text { Highly } \\
\text { recommended }\end{array}$ & Recommended & $\begin{array}{l}\text { Highly } \\
\text { recommended }\end{array}$ & Good \\
\hline MRC-D & Recommended & Recommended & $\begin{array}{l}\text { Highly } \\
\text { recommended }\end{array}$ & $\begin{array}{l}\text { Highly } \\
\text { recommended }\end{array}$ & $\begin{array}{l}\text { Highly } \\
\text { recommended }\end{array}$ & Good \\
\hline $\begin{array}{l}\text { RIQ- } \\
\text { MON10 }\end{array}$ & Recommended & Recommended & Good & Recommended & Insufficient & Insufficient \\
\hline SGRQ & $\begin{array}{l}\text { Highly } \\
\text { recommended }\end{array}$ & $\begin{array}{l}\text { Highly } \\
\text { recommended }\end{array}$ & Good & Good & Recommended & $\begin{array}{l}\text { Highly } \\
\text { recommended }\end{array}$ \\
\hline
\end{tabular}

Descrição e Limitações de algumas refermentas mais usadas ${ }^{35}$.

1- QUESTIONÁRIO DE VIAS AÉREAS (AQ20): se correlaciona bem com SGRQ e tem apenas 20 itens (sim / não). Curto e fácil de completar, necessitando em média em 2 minutos. Útil em um ambiente clínico. Auto administrado. Tem um menor poder discriminatório na DPOC leve. Tem versões em espanhol, japonês e português disponíveis. Interpretação: pontuação alta indica uma qualidade de vida ruim.

\section{2- PROBLEMAS DE RESPIRAÇÃO QUESTIONÁRIO} - BREVE (BPQ-S): Forma abreviada do BPQ. Não é específico para DPOC. Auto administrado, usado em DPOC leve a moderada. O BPQ-S é mais discriminatório para a DPOC do que a forma mais longa.

\section{3- ESCALA DE CLASSIFICAÇÃO DA ATIVIDADE DA} DPOC (CARS): Mede atividades relacionadas à vida na DPOC. Validade e confiabilidade testadas, mas poder discriminativo não foi testado. São analisados 4 fatores (autocuidado, atividades domésticas, atividades ao ar livre e interação social) com 12 itens. Escala fácil de três pontos. A literatura limitada, com poucos estudos disponíveis. Escores mais altos indicam menor comprometimento.

4- TESTE DE AVALIAÇÃO DA DPOC (CAT) Questionário curto e simples para monitorar o acompanhamento a longo prazo da DPOC. Dirigido à prática de cuidados primários. Validado em 3 estudos internacionais. Composto de 8 itens, e escala de seis pontos, identifica bem as exacerbações. Auto administrado. Publicado em 2009. Disponível em muitas traduções, embora nem todas validadas. Escores mais altos representam pior estado de saúde.

5- QUESTIONÁRIO DE COPD CLÍNICA (CCQ) bem validado e confiável. Bom para avaliar pacientes que estejam tentando parar de fumar e detecta estados leves de moderados e graves da doença. Mede capacidades funcionais e mentais, bem como sintomas. Específicos para DPOC. Auto administrado, bom para ser usado na prática diária. Composto de 10 itens sobre os sintomas da semana anterior, é fácil de aplicar. Também disponível em versão de avaliação de $24 \mathrm{~h}$. Prático e amplamente utilizado. Mais de 53 traduções: nem todas são validadas. Escores mais altos representam pior estado de saúde.

\section{6- QUESTIONÁRIO DE DOENÇAS RESPIRATÓRIAS} CRÔNICAS (CRQ) Bem validado e confiável. Revela as mudanças ao longo do tempo se usado a longo prazo, e identifica bem mudanças na condição após necessidade de tratamento de emergência motivado por exacerbações. Composto de 20 itens, e 4 domínios: Dispneia, fadiga, função emocional, domínio. Pode ser utilizado pelo entrevistador, por telefone ou ser auto administrado. Tem muitas traduções. Escores mais altos indicam melhor qualidade de vida relacionada à saúde. 
7- CONSELHO MÉDICO DE PESQUISA, de dispneia (MRC-D): Amplamente utilizado para avaliar como sintomas (dispneia) limita as atividades da vida diária. Bem validado. Cinco itens simples, mas mensura apenas medidas dispneia, não outros resultados. Tem versão Portuguesa validada no Brasil.

8- QUESTIONÁRIO DE MONITORIZAÇÃO DE 10 ITENS DE DOENÇA RESPIRATÓRIA - (RIQ-MON10: É uma redução da ferramenta QI RIQ de 55 itens. Bem validado, comparado com escalas SF-36 e MRC. É sensível à mudança em paciente estável e pacientes leves a moderados. Composto de 2 fatores (queixas físicas, mais emocionais e limitações físicas mais sociais) cinco itens cada. Não é específico para DPOC. Foi testado em cuidados primários.

\section{9- QUESTIONÁRIO DO HOSPITAL DE SAINT GE-} ORGE NA DOENÇA(SGRQ): É ○ instrumento de qualidade de vida mais utilizado na literatura, especialmente no domínio "sintomas", que pode ser usado sozinho. Comparado na literatura ao AQ20 e ao CRQ. É um padrão ouro, mas é muito longo, sendo assim sua aplicação não é simples. Duração de 8-10 minutos para ser concluído. As pontuações são calculadas para três domínios: sintomas, atividade e impacto (psicossocial), e também para pontuação total. Pode ser usado por telefone ou auto administrado. É sensível às mudanças na condição do paciente. Tem muitas traduções. Não é específico para DPOC. Os escores variam de 0 a 100, com escores mais altos indicando uma saúde precária.

\section{Conclusões}

A avaliação da qualidade de vida relacionada à saúde deve ser incorporada ao seguimento clínico em portadores de DPOC, uma vez que esta doença repercute nas diversas dimensões da vida dos pacientes. Os questionários específicos de QVRS parecem mensurar melhor o impacto da DPOC nas condições de saúde, permitindo a quantificação tanto no impacto da doença quanto no benefício das diversas intervenções disponíveis ou em teste atualmente na literatura científica.
Com o crescente interesse no conceito de qualidade de vida por pesquisadores das ciências médicas e por profissionais da assistência direta ao paciente, as escolas de saúde devem incluir em suas disciplinas o domínio do conhecimento de uso de QVRS, desde a escolha de questionários a serem utilizados até os métodos de aplicação dos mesmos nas diversas áreas. A apresentação da ferramenta questionário de qualidade de vida no período de formação acadêmica contribuirá para incorporar o seu uso na pratica médica.

\section{Contribuições dos autores}

Carneiro ACC participou da concepção, delineamento, busca dos dados da pesquisa, interpretação dos resultados, redação e encaminhamento do artigo científico. Costa, MC participou da coleta e interpretação dos dados. Costa, DMF participou da coleta e interpretação dos dados. Carneiro LC participou da coleta e interpretação dos dados e encaminhamento do artigo científico. Camelier, A participou da concepção, delineamento, busca dos dados da pesquisa, interpretação dos resultados, redação do artigo científico.

\section{Conflitos de interesses}

Nenhum conflito financeiro, legal ou político envolvendo terceiros (governo, empresas e fundações privadas, etc.) foi declarado para nenhum aspecto do trabalho submetido (incluindo mas não limitandose a subvenções e financiamentos, conselho consultivo, desenho de estudo, preparação de manuscrito, análise estatística, etc).

\section{Referências}

1. Vogelmeier CF, Criner GJ, Martinez FJ, Anzueto A, Barnes PJ, Bourbeau J et al. Global Strategy for the Diagnosis, Management, and Prevention of Chronic Obstructive Lung Disease 2017 Report. GOLD Executive Summary. Am J Respir Crit Care Med. 2017;195(5):557-582. doi: 10.1164/ rccm.201701-0218PP

2. OPAS/OMS Brasil. Doenças Respiratórias Crônicas [Internet]. [acesso em 2017 jun. 11]. Disponível em: http:// www.paho.org/bra/index.php?option $=$ com_content $\&$ view $=$ ar ticle\&id $=581$ : doencas-respiratorias-cronicas\&ltemid $=539$

3. Jones PW. Health status measurement in chronic obstructive pulmonary disease. Thorax. $2001 ; 56(1): 880-887$. doi: 10.1136/thorax.56.11.880 
4. Koskela J, Kilpelainen M, Kupiainen $H$, Mazur W, Sintonen $\mathrm{H}$, Boezen $M$ et al. Co-morbidities are the key nominators of the health related quality of life in and moderate COPD. BMC Pulm Med. 2014;14:102. doi: 10.1186/1471-246614-102

5. Jones PW, Kaplan RM. Methodological issues in evaluating measures of health as outcomes for COPD. Eur Respir J. 2003;21 (supl 41 ):13s-18s. doi: $10.1183 / 09031936.03 .00077802$

6. Smith KW, Avis NE, Assmann SF. Distinguishing between quality of life and health status im quality of life research: $A$ meta-analysis. Qual Life Res. 1999;8(5): 447-59.

7. Jones PW. Issues Concerning Health-Related Quality of Life in COPD. Chest. 1995;107(supl 5):187s-193s.

8. World Health Organization Quality of Life Assessment (WHOQOL): development and general psychometric properties. Soc Sci Med. 1998;46(12): 1569-85.

9. Scliar M. História do Conceito de Saúde. PHYSIS: Rev. Saúde Coletiva. 2007;17(1):29-41. doi: 10.1590/S0103$\underline{73312007000100003}$

10. Brasil. Constituição (1988). Constituição da República Federativa do Brasil. Brasilia, DF: Senado Federal,1988.

11. Vaitsman J, Andrade GRB. Satisfação e responsividade: formas de medir a qualidade e a humanização da assistência à saúde. Cienc Saúde Coletiva. 2005; 10(3):599-613. doi: $10.1590 /$ S1413-81232005000300017

12. Cohen $C$. On the quality of life: some philosophical reflections. Circulation 1982;66(supl III):29-33.

13. Diniz DP. Guia de qualidade de vida: saúde e trabalho. 2.ed. Barveri, SP: Manole; 2013.

14. Hajiro KT, Nishimura K. Minimal clinically significant difference in health status: the thornypath of health status measures? Eur Respir J. 2002;19:390-391.

15. Cheung Y-B, Goh C, Thumboo J, Khoo K-S, Wee J. Variability and Sample Size Requirements of Qualityof-Life Measures: A Randomized Study of Three Major Questionnaires. J Clin Oncol. 2005;23(22):4936-4944. doi: 10.1200/JCO.2005.07.141

16. Bouchet C, Guillemin F, Hoang Thi TH, Cornette A, Briançon S. Validation of the St George's questionnaire for measuring the quality of life in patients with chronic obstructive pulmonary disease. Rev Mal Respir. 1996;13(1):43-6.
17. Herdman $M$. The measurement of health related quality of life. Med Clin (barc) 2000; 114 (supl 3):22-5.

18. Benito CS. Medición de la calidad de vida: żcuestionarios genéricos o específicos? Measuring Quality of Life: Generic or Specific Questionnaires? España Arch Bronconeumol. 2005;41(3):107-9. doi: $10.1157 / 13071579$

19. American Thoracic Society. Chronic Obstructive Pulmonary Disease [Internet]. [acesso em 26 maio 2017]. Disponível em: http://qol.thoracic.org/sections/specific-diseases/copd.html

20. Mazur W, Kupiainen $H$, Pitkäniemi J, Kilpeläinen $M$, Sintonen $H$, Lindqvist $A$ et al. Comparison between the disease-specific Airways Questionnaire 20 and the generic 15D instruments in COPD. Health Qual Life Outcomes. 2011 ;9:4. doi: $10.1186 / 1477-7525-9-4$

21. Harper R, Brazier JE, Waterhouse JC, Walters SJ, Jones NM, Howard P. Comparison of outcome measures for patients with chronic obstructive pulmonary disease (COPD) in an outpatient setting. Thorax. 1997;52(10):879-87.

22. Tsukino $M$, Nishimura $K$, McKenna SP, Ikeda A, Hajiro T, Zhang $M$ et al. Change in generic and disease-specific healthrelated quality of life during a one-year period in patients with newly detected chronic obstructive pulmonary disease. Respiration. 2002;69(6):513-20. doi: 10.1159/000066456

23. Spencer S, Jones PW, GLOBE Study Group. Time course of recovery of health status following an infective exacerbation of chronic bronchitis. Thorax 2003;58(7):58993.

24. Domingo-Salvany A, Lamarca R, Ferrer M, GarcíaAymerich J, Alonso J, Félez $M$ et al. Health-related quality of life and mortality in male patients with chronic obstructive pulmonary disease. Am J Respir Crit Care Med. 2002; 166 (5):680-5. doi: $10.1164 / \mathrm{rccm} .2112043$

25. Sullivan $M$. The art of reading an article on quality of life research. New check list available to facilitate a critical evaluation. Lakartidningen. 2002;99(26-27): 2933-8.

26. Engström CP, Persson LO, Larsson S, Sullivan M. Healthrelated quality of life in COPD: why both disease-specific and generic measures should be used. Eur Respir J. $2001 ; 18(1): 69-76$.

27. Jones PW, Brusselle G, Dal Negro RW, Ferrer $M$, Kardos $P$, Levy $M L$ et al. Health-related quality of life in patients by COPD severity within primary care in Europe. Respir Med. 2011 ; 105(1):57-66. doi: 10.1016/i.rmed.2010.09.004 
28. Camelier A, Rosa F, Jones P, Jardim JR. Validação do questionário de vias aéreas 20 ("Airways questionnaire 20" - AQ20) em pacientes portadores de doença pulmonar obstrutiva crônica (DPOC) no Brasil. J Pneumol. 2003;29(1): 28-35.

29. Camelier A, Rosa FW, Nascimento OA, Fernandes ALG, Jardim JR. Propiedades discriminativas y validez de un cuestionario de salud en pacientes con enfermedad obstructiva de la vía respiratoria: el Airways Questionnaire 20 (AQ20). Arch Bronconeumol. 2007;43(1 2):662-8.

30. Sanchez FF, Faganello MM, Tanni SE, Lucheta PA, Padovani CR, Godoy I. Relationship between disease severity and quality of life in patients with chronic obstructive pulmonary Disease. Braz J Med Biol Res. 2008;41(10):860865. doi: $10.1590 /$ S0100-879X2008005000043

31. Camelier A, Rosa FW, Salmi C, Nascimento OA, Cardoso F, Jardim JR. Avaliação da qualidade de vida pelo Questionário do Hospital Saint George na Doença Respiratória em portadores de doença pulmonar obstrutiva crônica: validação de uma nova versão para o Brasil. J Bras Pneumol. 2006;32(2): 1 14-22. doi: 10.1590/S180637132006000200006

32. Sousa TC, Jardim JR, Jones P. Validação do Questionário do Hospital Saint George na Doença Respiratória (SGRQ) em pacientes portadores de doença pulmonar obstrutiva crônica no Brasil. J Pneumologia. 2000;26(3). doi: 10.1590/50102$\underline{35862000000300004}$

33. Kon SSC, Canavan JL, Jones SE, Nolan CM, Clark AL, Dickson $\mathrm{MJ}$ et al. Minimum clinically important difference for the COPD Assessment Test: a prospective analysis. Lancet Respir Med. 2014;2(3):195-203. doi: 10.1016/S22132600(14)70001-3

34. Jones PW, Price D, Van der Molen T. Role of clinical questionnaires in optimizing everyday care of chronic obstructive pulmonary disease. Int J Chron Obstruct Pulmon Dis. 2011 ;6:289-96. doi: 10.2147/COPD.S18181

35. Cave AJ, Atkinson L, Tsiligianni GL, Kaplan AG.

Assessment of COPD wellness tools for use in primary care: an IPCRG initiative. Int J Chron Obstruct Pulmon Dis. 201 2; 7:44756. doi: $10.2147 /$ COPD.S29868 